\title{
Letter
}

\section{Illumination to Assist Color Identification in Tritans}

\author{
Shigeharu TAMURA ${ }^{\dagger}$ and Yasushi SHIGERI
}

National Institute of Advanced Industrial Science and Technology (AIST), Japan

Received June 30, 2017, Accepted December 27, 2017

\begin{abstract}
We previously reported that light emitting diode (LED) light sources with longer wavelengths provided protans and deutans with greater red-green discrimination ability using a simulator and in volunteer subjects. Here, we focused on tritans, i.e., those with blue-yellow color blindness, and examined the effects of blue LEDs in a series of simulator studies. HRR pseudoisochromatic test plate No. 24 and SPP (standard pseudoisochromatic plates part 2) test plate No. 4 were illuminated by D65+LED $(470 \mathrm{~nm})$ or LED $(470 \mathrm{~nm})+\mathrm{LED}(590 \mathrm{~nm})$ and observed by a color vision simulator (chromatic vision simulator) that was produced on the basis of the Brettel's theory. These results indicated that blue LED light sources were effective for color discrimination of tritans.
\end{abstract}

KEYWORDS: aged person, HRR, LED, SPP, Vischeck

\section{Introduction}

As tritans (Type T) show reduced color discrimination ability between blue and yellow, they tend to confuse blue with yellow or orange with pink ${ }^{11}$. Recently, it was reported that $60 \%$ of aged people with no congenital color vision deficits showed similar symptoms to color blindness, and $80 \%$ of these people showed symptoms similar to those of tritans ${ }^{2}$. According to data of the Ministry of Internal Affairs and Communications, the population of people aged 75-84 in Japan, was 11,000,000 in 2013. About half of these people are assumed to be tritans. With the addition of elderly people older than 85 years old $(4,500,000)$, who have further possibility of color vision deficit, they far exceed the size of the population with congenital red-green color blindness in Japan (about 3,200,000). This may affect safety in daily life. The NHK (Japan's national public broadcasting corporation) TV program, "Tameshite Gatten", broadcast an episode entitled "Is this a mistake in vision? Real accidents caused by abnormalities of the eyes", on October 5th, 2016 ${ }^{3)}$. The TV program introduced some cases where blue socks were misread by aged people with color blindness and they had difficulty in identifying the blue flame of cooking devices. In addition, other cases were presented: (i) yellow implying danger was misread as achromatic colors, such as gray or white, or was not recognized; and (ii) blue that is used for ease of understanding by people with congenital color vision deficit was misjudged as gray or white.

Shionoda et al. reported that illumination with red light supported the color discrimination by protans and deutans who have difficulties in discriminating red and green, respectively ${ }^{4}$. Moreover, we reported previously that in the Ishihara pseudoisochromatic test and Farnsworth Panel D-15 test, LEDs with longer wavelength (red LEDs) were much more effective for improving color identification by deutans ${ }^{5-7)}$. Protans and deutans are less sensitive to red light than subjects with normal color vision and their relative visibility of red color is lower than that of green one $\left.(<10 \%)^{8}\right)$. On the other hand, tritans have impaired sensitivity to blue and typically show confusion between the colors blue and yellow ${ }^{1)}$. Taking the above phenomena into consideration, it is assumed that blue light would be effective to support color recognition by tritans. In this study, we performed a series of simulations of light source spectra to support discrimination of color patterns that are difficult for tritans to discriminate, and we discuss the role of blue LED light sources. A series of simulator studies were performed as tritans are extremely rare (less than 1 in 10,000) $)^{9}$.

\section{Experimental}

\subsection{Subjects}

Three adult male subjects $46-60$ years old $(46,54$, and 60$)$ and three female subjects 33-44 years old (33, 33, and 44) with normal color vision, participated in this study. All subjects were diagnosed in the Uzuki Clinic (Osaka, Japan) based on the Nagel type 1 anomaloscope (NEITZ, OT-II) and other methods, such as the HRR test (HRR Pseudoisochromatic Plates, Fourth Edition: Richmond Products Inc.) Nos. 22-24, SPP test (standard pseudoisochromatic plates part 2) No. 4 and the Farnsworth Panel D-15 test.

\footnotetext{
${ }^{\dagger}$ Corresponding Author: Shigeharu Tamura sg-tamura@aist.go.jp
} 


\subsection{Light sources and experiments}

Two types of blue light source were used: (A) CIE standard illuminant D65 (1501x)+LED (470 nm, 1501x) and (B) LED (470nm, 35lx)+LED (590 nm, 265lx). The total illuminance was $300 \mathrm{~lx}$. D65 was selected to mimic natural sunlight. An LED $(470 \mathrm{~nm})$ blue light source was utilized as red LED light sources were shown to be much more effective for improvement of color identification by deutans ${ }^{6}$. A combination of LED $(590 \mathrm{~nm})$ with LED $(470 \mathrm{~nm})$ was expected to produce white light. The spectral distributions of CIE standard illuminant D65 (150lx)+LED (470nm, 150lx) and LED (470 nm, 351x)+LED (590 nm, 265lx), observed using an illuminance spectrophotometer (CL-500 A; Konica Minolta, Tokyo, Japan) are shown in Figure 1. These illuminances were measured at the center of each chart. Each LED light source consisted of a 10×10 LED matrix and a diffusion panel (LSD10PC10; Optical Solutions, Tokyo, Japan) in front of the LED matrix. This system was set in a box containing a circuit board $(140(\mathrm{~W}) \times 85(\mathrm{D}) \times 55$ (H) $\mathrm{mm}$ ). The standard light source D65 was used to illuminate the observation object from $30^{\circ}$ above and in front at a distance of $50 \mathrm{~cm}$, while the LED light sources were placed in front of the observation object at a distance of $30 \mathrm{~cm}$ (Figure 2).

\subsection{Observation object}

Use of a color chart for color perception test was thought to be most appropriate as an example of an arrangement of colors that is difficult to distinguish regardless of color vision characteristics. In the HRR test, No. 24 containing symbols (cross and triangle), which was produced to identify tritan color perception, was used. Tritans could not recognize the cross symbol, while subjects with normal color vision could identify the symbol. In addition, the SPP test plate No. 4, which is read as " 67 " by subjects with normal color vision, was also utilized.

\subsection{Simulator}

Ideally, the effects of illumination to assist color identification in tritans should be verified by experiments with tritans. However, the total number of tritans in Japan is less than 5,000 and it is difficult to enroll such subjects diagnosed as tritans at healthcare facilities. Therefore, a color vision simulator (chromatic vision simulator) that was produced on the basis of the Brettel's theory ${ }^{10)}$ was utilized ${ }^{11)}$. This simulator operates on a tablet device, such as an iPad and can mimic the visual performance of protan (Type P), deutan (Type D), and tritan (Type T) color blindness. We observed the SPP test plate through the simulator in a tritan mode, changed the illumination and searched for the most suitable illumination conditions to allow tritans to discriminate patterns. The distance between the simulator

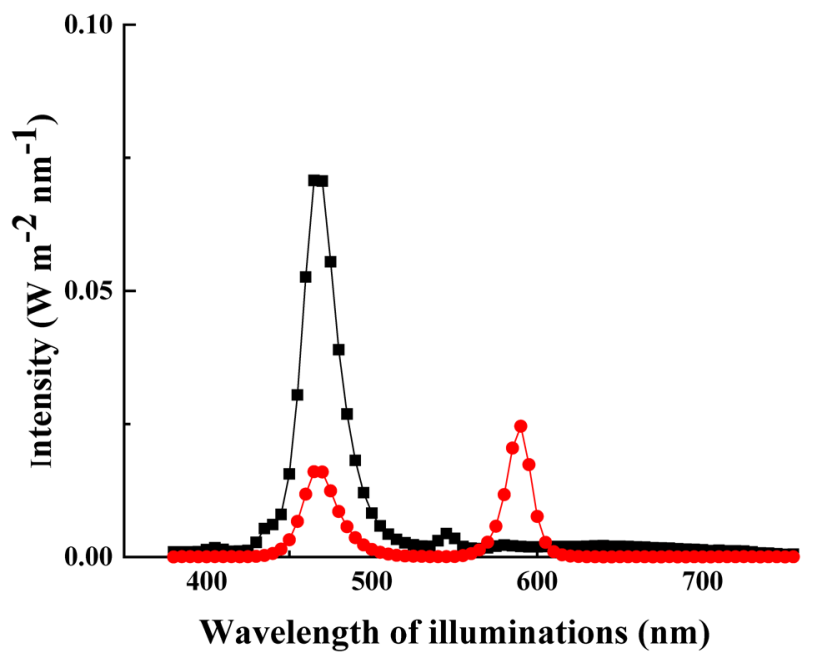

Figure 1 Spectral distributions of two illuminations, D65+LED $(470 \mathrm{~nm})$ and LED $(470 \mathrm{~nm})+$ LED $(590 \mathrm{~nm})$. Black squares and red circles are D65 (150lx)+LED (470 nm, $150 \mathrm{~lx})$ and LED (470 nm, 35Ix)+LED (590 nm, 265lx), respectively.

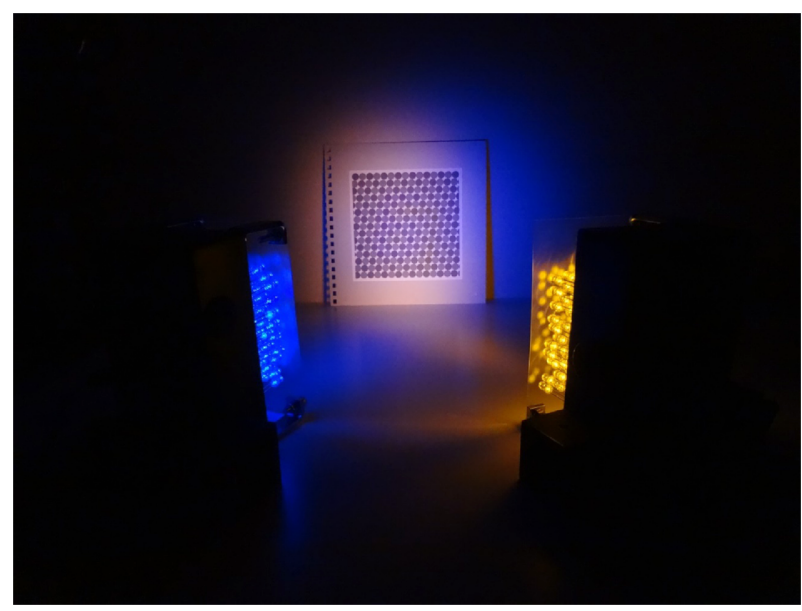

Figure 2 Experimental setup of test plates illuminated by two different LED light sources at wavelengths of 470 and $590 \mathrm{~nm}$.

and color chart was $75 \mathrm{~cm}$.

\section{Results and discussion}

3.1 Vision under conditions of CIE standard illuminant D65

We performed the observations of the HRR or SPP test plates that tritans can not generally read, in the normal color vision mode and tritan mode of the color vision simulator under D65 light (150lx). In tritan mode, neither the symbol (cross) in the HRR test plate nor letter in the SPP test plate could be observed, while subjects with normal color vision could recognize both plates. The HRR (symbols) and the SPP test (letters) plates in both normal color vision and tritan modes are shown in Figure 3. 
(a)

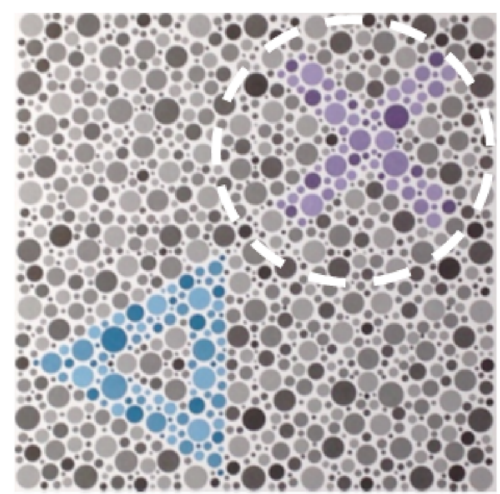

(c)

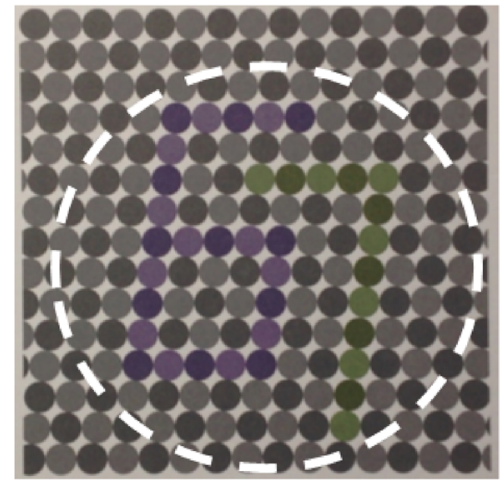

(b)

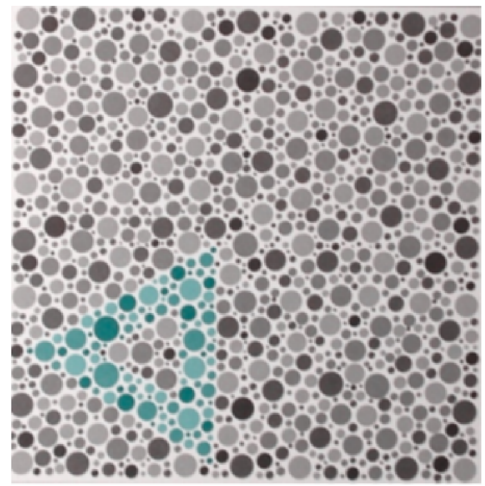

(d)

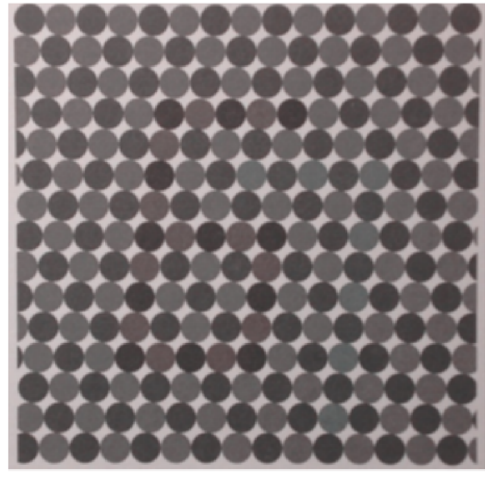

Figure 3 Simulation of HRR test (a, b) and SPP test plates (c, d) in normal color vision mode (a, c) and tritan mode (b, d) under the standard light source D65. The symbol or letter inside the white broken line could be observed in normal color vision mode.

\subsection{Standard light source D65 (150lx)+LED $(470 \mathrm{~nm}$, 1501x)}

Next, the HRR and SPP test plates were observed using the color vision simulator in normal color vision mode and tritan mode under D65 (150lx)+LED (470 nm, 150lx). Photographs of the HRR and SPP test plates in both normal color vision and tritan modes are shown in Figure 4. Due to the effect of LED $(470 \mathrm{~nm})$ illumination, the symbol (cross) in the HRR plate and the letter (67) in the SPP test plate could be recognized. The color rendering index of this light source was $\mathrm{Ra}=56.12$. We also utilized HRR No. 22 and No. 23 for the same experiments. Subjects answered correctly as well as in the case of HRR No. 24 .

\subsection{LED (470 nm, 351x)+LED (590 nm, 265 lx)}

Although the total illuminance of two LEDs was kept at $300 \mathrm{~lx}$, we searched for the most appropriate conditions under which the color plates could be recognized in the tritan mode. The best results were obtained with (LED $470 \mathrm{~nm}, 35 \mathrm{~lx}+\mathrm{LED} 590 \mathrm{~nm}, 265 \mathrm{~lx}$ ). Photographs of the HRR and SPP test plates under both normal color vision and tritan modes with the two LEDs $(470 \mathrm{~nm}$, $351 \mathrm{x}+590 \mathrm{~nm}, 265 \mathrm{~lx}$ ) are shown in Figure 5. The symbol (cross) in the HRR and the letter (67) in the SPP test plate could be recognized in both modes, as well as in Figure 4. We also utilized HRR No. 22 and No. 23 for the same experiments. Consequently, subjects answered correctly as well as in the case of HRR No. 24. The color rendering index of the light source was $\mathrm{Ra}=-1.95$.

To further analyze this phenomenon, the $\mathrm{L}^{*}$ and $\mathrm{a}^{*}$ values of the CIELAB coordinates of No. 24 in the HRR test plate were calculated. Using Image J software (Image processing and analysis in Java) ${ }^{12}$, the RGB values of five dots in the No. 24 HRR test plate under D 65 or LED $(470 \mathrm{~nm}, 35 \mathrm{~lx})+\mathrm{LED}(590 \mathrm{~nm}, 265 \mathrm{~lx})$ light in tritan mode were calculated, as shown in Figure 6(a) and 6(b), respectively. Dots 1,2 , and 3 are representative of the background, while dots 4 and 5 are parts of symbols. The obtained RGB values were then converted to the $\mathrm{L}^{*}$ and $\mathrm{a}^{*}$ values, as described previously ${ }^{5)}$. As shown in Figure $6(\mathrm{c}), \mathrm{L}^{*}$ and $\mathrm{a}^{*}$ values for five dots of two symbols and three backgrounds were relatively far apart under LED (470nm, 351x)+LED (590 nm, 2651x) compared to the case of D65. Taken together, these results suggest that a blue LED light source, such as $470 \mathrm{~nm}$, was effective for color identification by tritans.

\section{Conclusion}

Here, two light source spectra were proposed to support discrimination of color patterns that are difficult for tritans to discriminate. Illumination of the color test plates with these light sources and observation with a color vision simulator in tritan mode confirmed that discrimination was possible. Both light sources (D65+470 nm LED, 
(a)

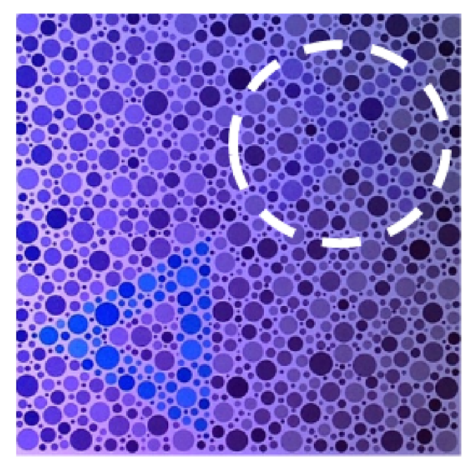

(c)

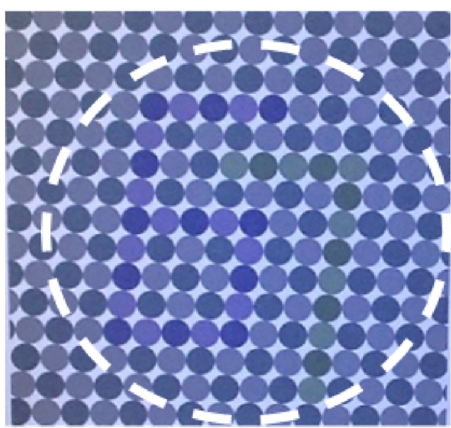

(b)

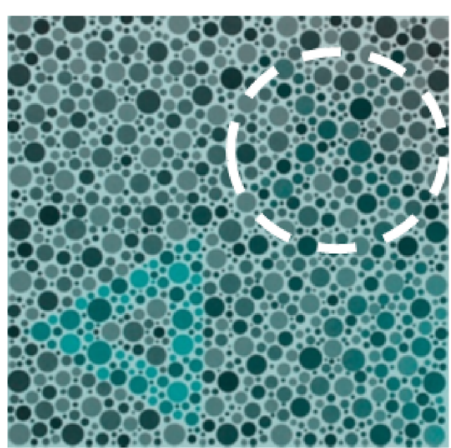

(d)

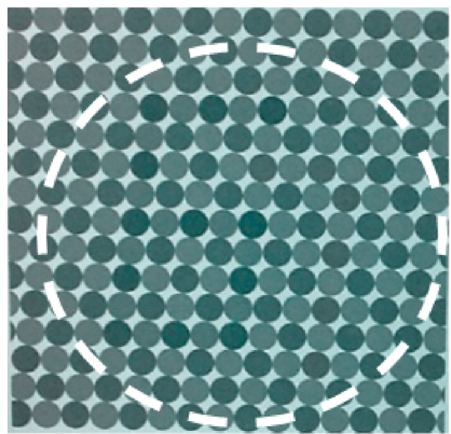

Figure 4 Simulation of HRR test $(a, b)$ and SPP test plates (c, d) in normal color vision mode $(a, c)$ and tritan mode (b, d) under the standard light source D65 (150 Ix)+LED ( $470 \mathrm{~nm}, 150 \mathrm{~lx})$ light. The symbol or letter inside the white broken line could be observed.

(a)

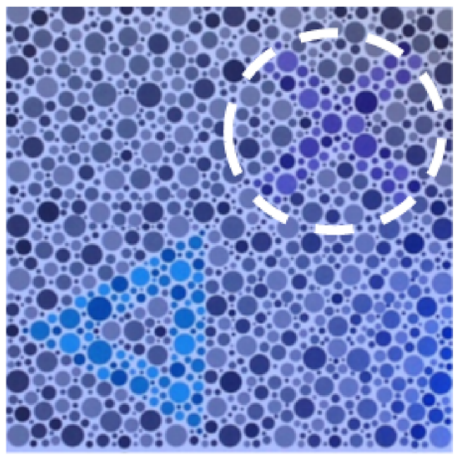

(c)

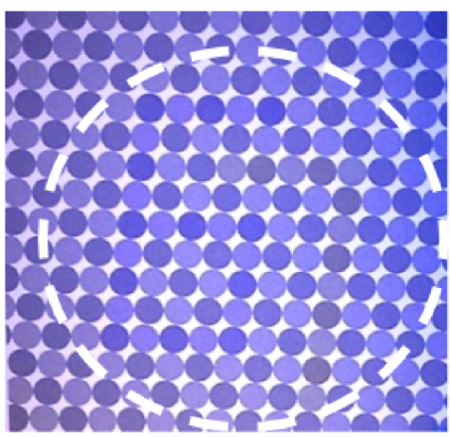

(b)

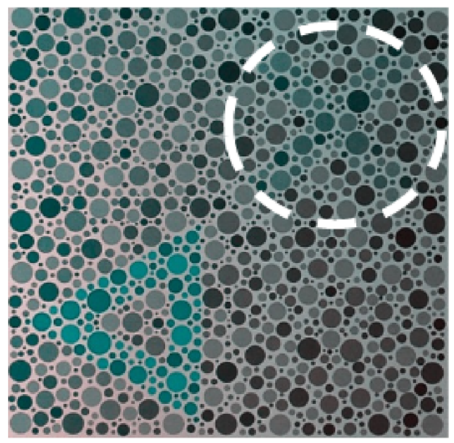

(d)

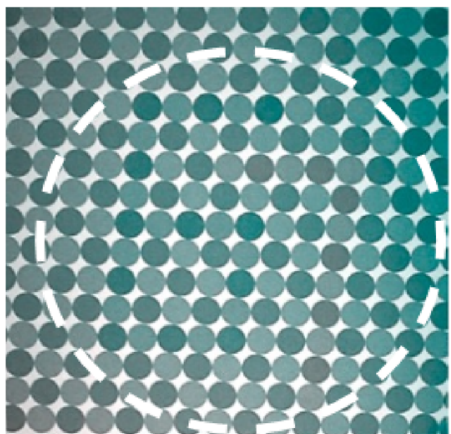

Figure 5 Simulation of HRR test ( $a, b)$ and SPP test plates (c, d) in normal color vision mode (a, c) and tritan mode (b, d) under LED $(470 \mathrm{~nm}, 35 \mathrm{~lx})+\mathrm{LED}(590 \mathrm{~nm}, 265 \mathrm{~lx})$. The symbol or letter inside the white broken line could be observed. 
(a)

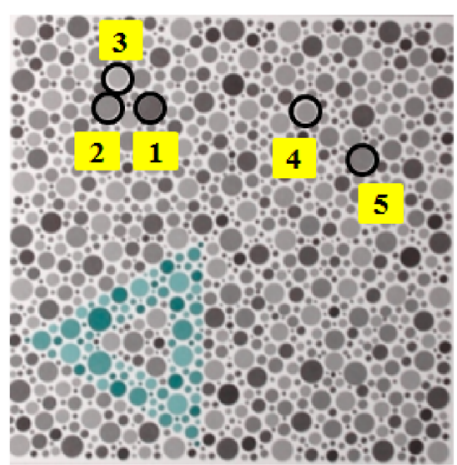

(c)

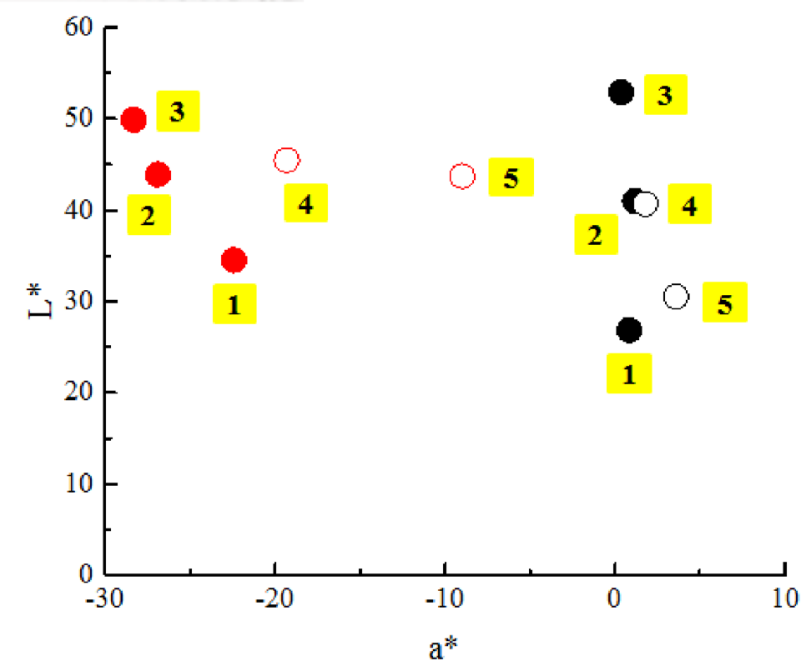

(b)

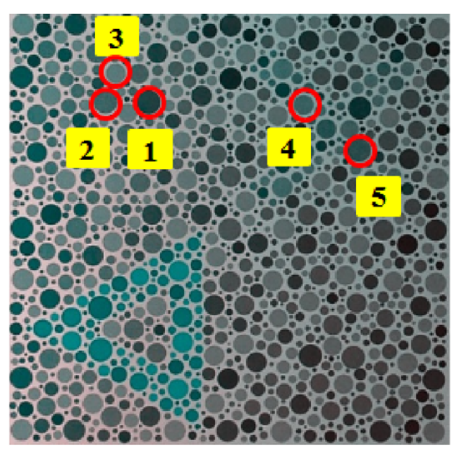
。

Figure 6 Positions of five dots in the No. 24 HRR test plate under the standard light source D65 (a) and LED (470 nm)+LED $\left(590 \mathrm{~nm}\right.$ ) light (b) in tritan mode. (c) Color information in the $\mathrm{L}^{*} \mathrm{a}^{*}$ plane obtained from five dots (two symbols and three background areas) in (a, black) and (b, red).

$470 \mathrm{~nm}$ LED+590 nm LED) proposed here, were shown to be effective for tritans with reduced blue-yellow discrimination ability by the combination of LEDs and simulator. Further studies are required to identify light sources that can be used to improve color discrimination ability by tritans, protans, and deutans, such as combinations of LEDs with wavelengths of $470 \mathrm{~nm}$ and $605 \mathrm{~nm}$.

\section{Acknowledgments}

This research was supported by LIXIL JS Foundation (15-14), Foundation for Promoting Personal Mobility and Ecological Transportation, and JSPS KAKENHI Grant Number 26350693 (to S. Tamura and Y. Shigeri).

\section{References}

(1) McIntyre, D.: Colour Blindness: Causes and Effects, Dalton Publishing, pp. 1-163 (2002).

(2) Schneck, M. E., Haegerstrom-Portnoy, G., Lott, L. A. and Brabyn, J. A.: Comparison of Panel D-15 tests in a large older population, Optom. Vis. Sci., 91-3, pp. 284-290 (2014).

(3) http://www9.nhk.or.jp/gatten/articles/20161005/ index.html.
(4) Shinoda, H. and Kanamori, A.: Lighting system for colorblind person, JP2008047465 (2006).

(5) Tamura, S., Okamoto, Y., Nakagawa, S., Sakamoto, T. and Shigeri, Y.: Practical color barrier-free illumination for deuteranopia using LEDs, Color Res. Appl., 40-3, pp. 218-223 (2015) .

(6) Tamura, S., Okamoto, Y., Nakagawa, S., Sakamoto, T., Ando, M. and Shigeri, Y.: Light wavelengths of LEDs to improve the color discrimination in Ishihara test and Farnsworth Panel D-15 test for deutans, Color Res. Appl., 42-4, pp. 424-430 (2017).

(7) Tamura, S. and Shigeri, Y.: Current status of light sources to assist color identification of congenital color blindness, J. Color Sci. Assoc. Jpn., 40, pp. 167-172 (2016).

(8) Peter, K. K. and Robert, MB.: Human Color Vision, Washington, DC: Optical Soc. Am., pp. 121-248 (1996).

(9) Boynton, R. M.: Human Color Vision, Holt, Rinehart and Winston, pp. 337-389 (1979).

(10) Brettel, H., Vienot, F. and Mollon, J. D.: Computerized simulation of color appearance for dichromats J. Opt. Soc. Am. A Opt. Image Sci. Vis., 14-10, pp. 2647-2655 (1997) .

(11) http://asada.tukusi.ne.jp/cvsimulator/e/index.html.

(12) https://imagej.nih.gov/ij/. 